\title{
Incidence of ventricular arrhythmias after left ventricular reconstructive surgery
}

\author{
Marisa Di Donato, MD, ${ }^{\mathrm{c}}$ Michel Sabatier, MD, ${ }^{\mathrm{a}}$ Lorenzo Menicanti, MD, ${ }^{\mathrm{b}}$ and Vincent Dor, MD ${ }^{\mathrm{a}}$
}

See related editorial on page 292.
From Centre Cardiothoracique de Monaco, ${ }^{\mathrm{a}}$ Monaco; San Donato Hospital, ${ }^{\mathrm{b}}$ Milan, Italy; and the Department of Critical Care Medicine, ${ }^{\mathrm{c}}$ University of Florence, Florence, Italy.

Received for publication July 13, 2006; accepted for publication Aug 7, 2006.

Address for reprints: Marisa Di Donato, MD, Department of Critical Care Medicine, University of Florence, Via dei Fossi 12, Florence 50123, Italy (E-mail: marad@ tin.it).

J Thorac Cardiovasc Surg 2007;133:289-91

$0022-5223 / \$ 32.00$

Copyright (C) 2007 by The American Association for Thoracic Surgery

doi:10.1016/j.jtcvs.2006.08.088
A recent article from O'Neill and coworkers ${ }^{1}$ published in the Journal in November 2005 reports that after left ventricular reconstruction (LVR) for ischemic cardiomyopathy, either akinetic or dyskinetic, patients might remain at risk for malignant ventricular arrhythmias and hence might benefit from a prophylactic implantable cardioverter defibrillator (ICD). There are no clear guidelines regarding the necessity of early ICD implantation in patients undergoing LVR because the most important trials addressing this issue excluded patients within 3 months (Madit II) after coronary artery bypass grafting surgery. ${ }^{2}$

The authors report a high residual incidence of inducible ventricular tachycardia (VT) after surgical intervention (42\%), and they conclude that either early ICD implantation or predischarge electrophysiologic (EP) study for risk stratification is indicated in patients submitted to LVR for ischemic dilated cardiomyopathy.

Major limitations of that study are that (1) ventricular volumes are not measured before and after, and (2) the EP study is performed only after surgical intervention, and this negates the possibility of evaluating the effects of LVR on arrhythmias.

The authors look at 217 patients who had LVR and divide them into 3 groups. Groups 1 and 2 had an ICD implanted either before or after surgical intervention. Thirty patients had ICDs in situ before the operation (group 1), and 74 patients had ICD implantation after LVR (group 2); indications for postoperative ICD implantation were secondary prevention in 28 and primary prevention in 48 (positive EP study result). The authors then compare these 2 groups with another group of 116 patients who did not receive an ICD. Of these, 67 had EP studies (negative results in 65), and 46 did not undergo EP studies for some reason.

Patients in group 1 are the sickest because they have the largest volumes, more frequent need of concomitant mitral valve surgery, and the lowest proportion of concomitant revascularization. Group 1 had the highest rate of events (progressive heart failure was the most frequent cause of death). Overall, the incidence of sudden death was extremely low, with only 1 sudden death of 20 deaths, which occurred in 1 patient who had ICD implantation before the operation. They compare mortality in the 3 groups and show that annualized mortality is significantly lower in groups 2 (with an ICD implanted for primary or secondary prevention) and 3 (without ICDs) with respect to group 1 (ICDs implanted before the operation). These groups are not comparable preoperatively, and we can presume that they are not comparable also after surgical intervention, but the authors do not provide postoperative data. We know that 15 patients with ICDs, 6 in group $1(20 \%)$ and 9 in group $2(12 \%)$, had appropriate ICD therapies, which saved their lives, according to the O'Neill interpretation. Independent predictors of ICD firings were preoperative volumes and ejection fraction (EF), and we can hypothesize that patients who received ICD therapy and those who had positive EP study results early after surgical intervention did not have an improvement in volumes and EF after LVR.

Ventricular volume before and after surgical intervention is crucial for arrhythmia development based on the following considerations. A large ventricular volume brings high wall stress and high stretch, and stretch is arrhythmogenic. ${ }^{3,4}$ In previous $\operatorname{articles}^{5,6}$ we have clearly demonstrated that patients with ventricular arrhythmias (spontaneous or inducible) have end-diastolic and end-systolic volumes significantly larger than those seen in noninducible patients and that patients who die at follow-up have the largest ventricular volumes. The antiarrhythmic effect of LVR has been 
demonstrated by several groups ${ }^{7,8}$ and is related to volume reduction (less tension and stretch) and to the exclusion of the myocardial scar, which constitutes the trigger for electrical instability, but also to complete revascularization, which relieves ischemia (another important component of electrical instability), and to mechanical resynchronization, ${ }^{9}$ which brings a more homogeneous distribution of wall tension and reduces regional prestretch. ${ }^{10}$ In contrast with studies that demonstrate the antiarrhythmogenic effect of LVR, the group from the Cleveland Clinic found a high incidence of residual inducible arrhythmias after reconstructive surgery, and they implanted an ICD in almost $50 \%$ of their series. We have different results because we have inducible VT in $45 \%$ of patients preoperatively $(75 \%$ in patients with spontaneous ventricular arrhythmias), and postoperatively (early and 1 year after surgical intervention), $90 \%$ of patients are free from inducible VT. Thus in our experience LVR shows a dramatic reduction in inducible arrhythmias, which is confirmed by the recent article by Sartipy and coworkers. ${ }^{7}$

Possible explanations for this discrepancy are as follows. First, the technique used is different from the Dor procedure, in which the use of the intraventricular patch is considered mandatory. In the Cleveland series the patch has not been used in most patients, and only $13 \%$ of the patients received cryoablation, whereas in the Dor series all patients with either inducible or spontaneous arrhythmias have total endocardectomy plus cryoablation at the border of the transitional zone. Second, the stimulation protocol differs: it is more aggressive at the Cleveland Clinic, with up to 4 extrastimuli at 2 ventricular sites versus up to 2 extrastimuli at 1 ventricular site in our center. Third, the authors do not report whether a significant volume reduction has been achieved. Finally, EP study is not performed before surgical intervention, and this negates the possibility of demonstrating the effects of LVR on ventricular arrhythmias.

The authors state that ICD implantation might have saved 15 lives because there were 15 appropriate ICD firings. The Madit II study showed that an appropriate firing of an ICD identifies patients at increased risk for subsequent heart failure and nonsudden cardiac death. ${ }^{3}$ O'Neill and coworkers ${ }^{1}$ have 6 appropriate ICD firings in group 1 (the sickest group) and 6 deaths, 5 caused by progressive heart failure. It would be interesting to know whether the patients who died in group 1 were those who had ICD firings early after surgical intervention. They do not have sudden death in the group without ICD; they interpret this as due to the negative EP study result, but first, the validity of the EP study as a risk stratifier is questionable, and second, almost half of the patients without ICDs did not have EP studies for some reason. We think that the good survival rate of the O'Neill population (90\% at 1 year) should be ascribed to surgical intervention independent of ICD implantation.
TABLE 1. Any cause mortality at follow-up (time from operation to death)

\begin{tabular}{cccc}
\hline & $\leq 3$ mo & $>3$ to $\leq 36$ mo & $>36$ mo \\
\hline No. of patients $(\%)$ & $22(10)$ & $101(46)$ & $98(44)$
\end{tabular}

The median time to first ICD therapy was 43 days. Most (67\%) first therapies occurred within the first 63 days. Therefore O'Neill and coworkers ${ }^{1}$ propose the strategy of early EP study, early ICD implantation, or both to save lives from sudden death. We looked at our global population and found that the majority of deaths occurred after 3 years from surgical intervention (mean, $68 \pm 22$ months) and that less than $10 \%$ occurred in the first 3 months (Table 1).

The MADIT II study showed that noninducible patients received more ICD shocks for ventricular fibrillation than did inducible patients, and we confirm this finding because in our series patients who died of sudden death were not inducible after LVR. ${ }^{6}$

Postoperative inducible VT occurs in a small proportion of our patients $(90 \%$ are free from inducible VT). In the early postoperative period, inducible VT can be ascribed to electrolytic abnormalities, tissue edema, or inflammation, and we prescribe amiodarone. If the EP study result is still positive at least 4 months after surgical intervention or if the patient has spontaneous malignant ventricular arrhythmias, ICD implantation is indicated. Frequently, patients with a positive EP study result early after surgical intervention have negative results late after surgical intervention.

LVR induces a significant improvement in EF, and patients who would have fit Madit II criteria before surgical intervention might no longer be ICD candidates. EF improves well beyond $30 \%$ (preoperative median, 29\%; postoperative median, $44 \%$ ); however, there is a small proportion of patients who continue to have EFs equal to or lower than $30 \%$ after surgical intervention, and we observed a higher mortality at follow-up in these patients. Thirty-four (7\%) of 464 patients had a postoperative EF of $30 \%$ or less; $8(23 \%)$ of them died at follow-up. We therefore would suggest waiting for postoperative pump function improvement before considering ICD implantation.

In our overall population of 1448 survivors, the rate of ICD implantation is around 5\%. Interestingly, 694 of 1448 patients were operated on at the cardiothoracic Center of Monaco (between 1989 and 2002) and had endocardectomy and cryoablation added to LVR if they had spontaneous arrhythmias, inducible arrhythmias, or both; 754 of 1448 patients were operated on in Milan (1989-2002) and did not receive cryoablation or EP study. Late mortality was $15 \%$ $(221 / 1448)$ at $41 \pm 38$ months, without significant differences between the 2 centers; the rate of ICD implantation is similar in the 2 centers. This suggests that the high electrical success rate is mainly attributable to reduction of left ven- 
tricular volume with septal exclusion, complete revascularization, and scar resection, which are common procedures in both centers, rather than to the large encircling cryoablation, which is performed only for the Dor procedure. All those mechanisms, including mechanical resynchronization, might act to prevent functioning of the re-entry circuit and to reduce stretch-induced arrhythmias.

In conclusion, the article from O'Neill and coworkers ${ }^{1}$ addresses an important issue in the use of ICDs after LVR. We think that more studies and larger experience are needed to provide a correct indication for ICD in patients with dilated ischemic cardiomyopathy submitted to volume reduction surgery.

\section{References}

1. O’Neill JO, Starling RC, Khaykin Y, et al. Residual high incidence of ventricular arrhythmias after left ventricular reconstructive surgery. J Thorac Cardiovasc Surg. 2005;130:1250-6.

2. Moss AJ, Greeberg H, Case RB, et al. Long term clinical course of patients after termination of ventricular tachyarrhythmias by an implanted defibrillator. Circulation. 2004;110:3760-5.
3. Koilpillai C, Quinones MA, Greenberg B, et al. Relation of ventricular size and function to heart failure status and ventricular dysrhythmia in patients with severe left ventricular dysfunction. Am J Cardiol. 1996; 77:606-11.

4. Babuty D, Lab MJ. Mechanoelectric contributions to sudden cardiac death. Cardiovasc Res. 2001;50:270-9.

5. Di Donato M, Sabatier M, Dor V, the RESTORE group. Surgical ventricular restoration in patients with post-infarction coronary artery disease: effectiveness on spontaneous and inducible ventricular tachycardia. Semin Thorac Cardiovasc Surg. 2001;13:480-5.

6. Di Donato M, Sabatier M, Dor V, Buckberg G, RESTORE Group. Ventricular arrhythmias after LV remodelling: surgical ventricular restoration or ICD? Heart Fail Rev. 2004;9:299-306.

7. Sartipy U, Albage A, Straat E, et al. Surgery for ventricular tachycardia in patients undergoing left ventricular reconstruction by the Dor procedure. Ann Thorac Surg. 2006;81:65-71.

8. Mikleborough LL, Merchant N, Ivanov J, Rao V, Carson S. Left ventricular reconstruction: early and late results. $J$ Thorac Cardiovasc Surg. 2004;128:27-37.

9. Di Donato M, Toso A, Dor V, et al. Surgical ventricular restoration improves mechanical intraventricular dyssynchrony in ischemic cardiomyopathy. Circulation. 2004;109:2536-43.

10. Breithardt OA, Stekllbrink C, Herbots L, et al. Cardiac resynchronization therapy can reverse abnormal myocardial strain distribution in patients with heart failure and left bundle branch block. J Am Coll Cardiol. 2003;42:486-94. 